\title{
Psicologia da amizade na infância: uma revisão crítica da literatura recente
}

\author{
Agnaldo Garcia \\ Universidade Federal do Espírito Santo
}

\begin{abstract}
RESUMO
Este artigo apresenta uma revisão crítica da literatura recente sobre os aspectos psicológicos da amizade na infância. Aproximadamente, uma centena de artigos publicados em revistas indexadas de circulação internacional, de 1995 a 2003, foi selecionada e analisada. Os temas encontrados foram organizados em quatro grupos: 1) Aspectos metodológicos; 2) Dimensões de análise (incluindo similaridade e simetria, processos de cooperação, competição, apoio social, conflito e agressividade); 3) Redes sociais (a criança, seus pares e amigos) e contexto (família e escola); 4) Casos especiais: deficiência física e mental, rejeição, negligência, abuso, depressão e solidão. A literatura analisada apresenta: a) orientações teóricas e estratégias metodológicas diversificadas; b) falta de modelos teóricos da amizade ou de seus aspectos; c) carência de investigações em países em desenvolvimento. São propostas algumas possíveis orientações para a pesquisa empírica e teórica, incluindo a investigação das relações de amizade atribuídas aos familiares, novas dimensões da amizade na escola e na vizinhança, ampliação no número e na profundidade das dimensões investigadas, além da ampliação de casos especiais a serem considerados nos estudos da amizade, incluindo portadores de necessidades especiais e estudos sobre crianças enfrentando diferentes situações estressantes, como enfermidade física ou problemas psicológicos.
\end{abstract}

Palavras-chaves: amizade; infância; relacionamento interpessoal.

\begin{abstract}
The psychology of friendship in childhood: a critical review of the recent literature

This article presents a critical review of the recent literature about psychological aspects of friendship in childhood. A hundred articles, approximately, published in international indexed journals, from 1995 to 2003, have been selected and discussed. The themes present in these texts were organized in four thematic groups: 1) Methodological aspects; 2) Dimensions of analysis (including similarity and symmetry, processes of cooperation, competition, social support, conflict and aggressiveness); 3) Social networks (the child, its peers and friends) and context (family and school); 4) Special cases: physical and mental disability, rejection, negligence, abuse, depression and loneliness. The literature analyzed presents: a) diversified theoretical orientations and methodological strategies; b) lack of theoretical models of friendship or its aspects; c) lack of investigations in developing countries. Some possible empirical and theoretical research directions are proposed, including the investigation of friendship relations attributed to relatives, new dimensions of friendship at school and in the neighborhood, the increase in the number (and depth) of the investigated dimensions, besides the widening of the special cases to be considered in the studies about friendship, including disabled children and also children facing different stressful situations, such as physical disease or psychological problems.
\end{abstract}

Keywords: friendship, childhood, personal relationships.

Ao lado do relacionamento romântico e das relações familiares, a amizade representa, atualmente, uma das formas de relacionamento mais investigadas, passando a ser tema de investigação sistemática a partir dos anos 80. Ainda assim, a amizade permanece a menos investigada das três áreas, carecendo de um maior número de pesquisas teóricas e empíricas, o que pode ser percebido por uma certa estabilidade no número de publicações sobre os aspectos psicológicos da amizade na infância ao longo dos últimos anos. A amizade também tem se tornado uma área autônoma de investigação, merecedora de elaborações teóricas e 
procedimentos metodológicos particulares. A amizade na infância, ao lado da aceitação pelos pares, representa uma importante forma de socialização, com características próprias e uma literatura específica, justificando os esforços para a construção de modelos teóricos para as relações de amizade, como uma subárea de uma ciência dos relacionamentos (Hinde, 1997). Do ponto de vista social, as transformações da sociedade contemporânea ocidental têm exposto as crianças cada vez mais cedo e mais intensamente ao contato com os pares, fora da família. Os contatos mais intensos, provocados pela escolarização mais precoce, pela urbanização, pela redução da família, possivelmente representam fatores que contribuem para a importância crescente das relações de amizade na vida da criança.

O objetivo do presente trabalho é efetuar uma revisão crítica de uma seleção de artigos sobre os aspectos psicológicos da amizade na infância, publicados entre 1995 e 2003 (inclusive), em revistas científicas de circulação internacional, indexadas pela American Psychological Association, visando contribuir para a definição e a delimitação de uma "Psicologia da Amizade na Infância", associando-se ao esforço de outros autores (como Hinde, 1997) e mesmo de sociedades científicas internacionais (como a International Association for Relationship Research), no sentido de promover o avanço na organização teórica de pesquisas sobre o relacionamento interpessoal. Para a realização deste trabalho, foram selecionados artigos tendo a amizade na infância como tema central. Após a identificação destes textos, foram selecionados os artigos publicados em periódicos com pelo menos duas publicações com as características acima, ao longo do tempo. Os temas tratados nas publicações selecionadas foram identificados e organizados em grupos, visando fornecer um panorama das pesquisas na área nos últimos anos. Os temas identificados foram reunidos em quatro grupos, propostos a partir da análise do conteúdo desses artigos: a) aspectos metodológicos (artigos discutindo a metodologia aplicada a pesquisas sobre a amizade na infância); b) dimensões de análise (estudos sobre aspectos internos das relações de amizade, incluindo os processos psicossociais investigados); c) amizades, redes sociais e contexto (artigos abordando aspectos externos das amizades, situando-as em seu contexto social); e d) casos especiais de amizade (artigos sobre as amizades de crianças com necessidades educativas especiais).

Tendo em vista a constituição de uma "Psicologia da Amizade na Infância", nas considerações finais, são apresentadas algumas possibilidades de avanço empírico e teórico, visando contribuir para a consoli- dação desta área de investigação como uma sub-área de uma ciência mais ampla do relacionamento interpessoal.

\section{Aspectos Metodológicos}

A autonomia da amizade como área de estudos encontra sua base não apenas no reconhecimento da especificidade de suas propriedades e na construção de um corpo teórico específico, mas também em uma literatura que já trata especificamente da metodologia de estudo sobre a amizade, no presente caso, particularmente no período da infância.

Apesar dos estudos sobre a amizade representarem apenas parte dos estudos sobre as relações sociais na infância, um número significativo de trabalhos discute especificamente aspectos metodológicos da pesquisa sobre a amizade nesse período. Estes trabalhos evidenciam a tendência para a diversidade ou pluralidade metodológica. As discussões metodológicas incluem o desenvolvimento de escalas para medir pontos específicos (Rotenberg \& Morgan, 1995), a validação de instrumentos de pesquisa (Camaioni, Baumgartner \& Perugini, 1998), aspectos psicométricos e sociométricos ligados à metodologia (Erdley, Nangle \& Gold, 1998) e formas de medir as amizades (Schneider \& Greenman, 2003). Diversas técnicas têm sido propostas na investigação da amizade na infância, como a utilização de histórias (Smorti \& Pagnucci, 2003), de desenhos (Bombi, Cannoni \& Pinto, 2003) e de grades de observação (Baumgartner \& Camaioni, 1995). Face à diversidade de procedimentos metodológicos sobre a amizade e outras relações com pares na infância, alguns trabalhos específicos têm apresentado uma revisão da metodologia (Yugar \& Shapiro, 2001).

As diversas propostas metodológicas presentes na literatura se apóiam em diferentes orientações teóricas. De forma geral, há uma tendência para a diversificação metodológica como reflexo da pluralidade teórica. A busca de uma "ciência do relacionamento da amizade", como subárea de uma "ciência do relacionamento interpessoal" representa um desafio, no sentido de buscar integrar contribuições de diferentes origens teóricas, com reflexos nos procedimentos metodológicos.

\section{Dimensões de Análise}

A pesquisa sobre a amizade na infância utiliza algumas dimensões tipicamente empregadas nos estudos sobre o relacionamento interpessoal, como similaridade, simetria, cooperação, apoio social, competição, conflito e agressividade. Estas, por vezes, são 
reunidas em dois grupos opostos: o lado positivo do relacionamento (light side), incluindo dimensões como cooperação e apoio social, e o negativo (dark side), reunindo dimensões como conflito e agressividade.

Simetria e, particularmente a similaridade, são duas dimensões centrais nos estudos sobre a amizade. A similaridade é reconhecida como um traço fundamental dessa forma de relacionamento (Haselager, Hartup, Lieshout \& Riksen-Walraven, 1998; French, Jansen, Riansari \& Setiono, 2003) e inclui semelhanças em características demográficas (como idade e gênero), no status sociométrico, no comportamento agressivo e desempenho acadêmico, as quais servem de base para as amizades (Kupersmidt, DeRosier \& Patterson, 1995), o mesmo ocorrendo com similaridade comportamental (Poulin \& cols, 1997), inclusive entre crianças de diferentes países (Pinto, Bombi \& Cordioli, 1997). A simetria é outra dimensão da amizade (Bensalah, 1995), contudo, é possível encontrar relações assimétricas, especialmente entre crianças com idades diferenciadas. Outras dimensões menos investigadas são a influência social (Hawley, Little \& Pasupathi, 2002) e a intimidade e auto-revelação (Rotenberg, 1995), mais evidentes na adolescência, mas já ocorrendo na infância.

Algumas dimensões se referem a processos psicossociais entre amigos, como cooperação, competição e apoio social. A cooperação entre amigos estimula a criatividade (Miell \& MacDonald, 2000), como no caso da criação musical. A contraposição, contudo, também faz parte das relações de amizade (Tomada, 2000) e amigos competem mais acirradamente em jogos competitivos que não amigos (Fonzi, Schneider, Tani \& Tomada, 1997). A competição entre amigos, todavia, também pode dar lugar a formas negativas de comportamento (Hughes, Cutting \& Dunn, 2001). A amizade também influencia o comportamento prósocial demonstrado em relação à outra criança, predispondo-a à ação (Sebanc, 2003). O comportamento pró-social (incluindo o cooperar e o compartilhar) tem sido um dos aspectos mais claramente associados à amizade. O papel da competição entre amigos é mais controverso, mas a literatura tem reconhecido a presença da competição entre amigos.

Amigos e familiares oferecem formas diferentes de apoio social na infância. Os amigos são uma importante fonte de apoio emocional (Prinstein, La-Greca, Vernberg \& Silverman, 1996; Booth, Rubin \& RoseKrasnor, 1998), contribuindo para a redução de estresse no enfrentamento de preocupações compartilhadas (Rizzo \& Corsaro, 1995). O apoio social provido por amigos apresenta diferenças culturais, sendo maior em alguns países, especialmente no Ocidente. Em diferentes culturas, contudo, amigos são fontes importantes de intimidade e companheirismo (French, Rianasari, Pidada, Nelwan \& Buhrmester, 2001).

Conflito e agressividade integram as relações de amizade. Amigos agem de forma diferenciada de não amigos na negociação de conflitos, fazendo mais propostas ou negociando mais (Fonzi \& cols., 1997). Por outro lado, a forma como a criança lida com o conflito também afeta a amizade. A intenção de vingança em resposta a conflito com um amigo, por exemplo, está associada com a falta de amigos ou com amizades de baixa qualidade (Rose \& Asher, 1999). A agressividade, de modo geral, é percebida pelas crianças como um fator de distanciamento, dificultando o estabelecimento de amizades. Quanto mais agressiva a criança, menor sua aceitação social pelos colegas em termos de amizade (Phillipsen, Deptula \& Cohen, 1999). Por outro lado, há correlações positivas de comportamentos agressivos entre crianças populares (e de popularidade média) e seus amigos mútuos, indicado que crianças agressivas podem se atrair mutuamente (Ray, Cohen, Secrist \& Duncan, 1997). A manifestação de fantasia violenta também estava relacionada a comportamento anti-social frequiente, mostras de raiva, conflito e recusa a ajudar um amigo (Dunn \& Hughes, 2001). Enquanto a agressividade, à primeira vista, cria barreiras para as relações de amizade, há formas de lidar com o comportamento agressivo dentro das relações de amizade (Brendgen, Vitaro, Turgeon \& Poulin, 2002).

Os aspectos cognitivos investigados nas relações de amizade se referem ao processamento de informação, desde a percepção social, expectativas, compreensão e conceito de amizade, até avaliação da relação com o amigo. A percepção social envolve a autopercepção e a percepção da própria habilidade de fazer amigos (Barry \& Wigfield, 2002), a percepção do amigo (Hortacsu, Gencoez \& Oral, 1995; Barry \& Wigfield, 2002) e da própria relação de amizade (Little, Brendgen, Wanner \& Krappmann, 1999; Sturgess, Dunn \& Davies, 2001; Weiss, Smith \& Theeboom, 1996; Ollendick, Yang, Dong, Xia \& Lin, 1995). Por outro lado, a condição de ser amigo, e suas características, ainda pode (ou não) afetar as cognições sociais (Brendgen, Bowen, Rondeau \& Vitaro, 1999). Outros aspectos cognitivos relacionados à amizade envolvem compreensão social e comunicação (Brown, DonelanMcCall \& Dunn, 1996; Maguire \& Dunn, 1997; Dunn \& Cutting, 1999; Dunn, Cutting \& Demetriou, 2000; Slomkowski \& Dunn, 1996; McElwain \& Volling, 2002), o uso de mapas com amigos mais jovens e mais velhos (Wallace \& Almy, 1999), o raciocinar da 
criança sobre a importância da amizade na escolha de um parceiro (Halle, 1999); o conceito e expectativas de amizade (Salisch \& Seiffge-Krenke, 1996; Ray \& Cohen, 1996); avaliações do relacionamento (Meurling, Ray \& LoBello, 1999); e aspectos morais (Dunn, Cutting \& Demetriou, 2000). Do lado afetivo, a amizade tem sido considerada importante para o desenvolvimento emocional (Salisch, 2001). Estes estudos enfatizam a cognição social, da percepção ao processamento de informações.

Alguns estudos privilegiam o desenvolvimento da amizade, sua dimensão temporal. As amizades apresentam uma fase inicial, de estabelecimento (com o processo de escolha de amigos), uma fase de manutenção (sujeita a alterações e diferentes níveis de estabilidade) e um possível término. Entre os fatores que influenciam a escolha de amigos, estão gênero e etnia (Graham \& Cohen, 1997; Graham, Cohen, Zbikowski $\&$ Secrist, 1998), sendo o gênero usualmente considerado a maior influência. Uma vez estabelecida a amizade, ela está sujeita a alterações. No caso de mudança de nível escolar pela criança, por exemplo, as amizades foram afetadas de forma diferente (Dunn, Cutting \& Fisher, 2002; Corsaro, Molinari, Hadley \& Sugioka, 2003). A estabilidade das amizades na infância tem, por vezes, suscitado discussões. Maior estabilidade tem sido atribuída a uma melhor qualidade de comunicação, facilitando a resolução de conflitos (Schneider, Fonzi, Tani \& Tomada, 1997). A duração da amizade afeta a satisfação com o relacionamento e amizades de curta duração parecem ser menos satisfatórias que as mais duráveis (Parker \& Seal, 1996). O término de uma amizade apresenta conseqüências positivas, como abrir novas possibilidades de relacionamento, e negativas, como o sentimento de perda e tristeza (Parker \& Seal, 1996).

\section{Amizades, Redes Sociais e Contexto}

As relações de amizade ocorrem dentro de redes sociais complexas, incluindo a criança (com suas características individuais), seus pares e amigos, além de familiares e outras pessoas. Dois contextos estão intimamente ligados às amizades: o familiar e o escolar.

A amizade representa uma forma particular de relacionamento com pares na infância que, por vezes, é comparada com outras formas distintas de relacionamento, como a aceitação social (Linsey, 2002; Gifford-Smith \& Brownell, 2003) e as relações com colegas (Hundley \& Cohen, 1999; Simpkins \& Parke, 2002). Há, contudo, uma tendência recente a destacar a amizade como um objeto diferenciado de investigação, um relacionamento particular com propriedades diferenciadas. As pesquisas sobre a amizade infantil cobrem desde aspectos pessoais da criança, seja daquela com muitos amigos (Tani, 2000) ou daquela sem amigos (Doll, 1996), até a natureza das redes de amigos. Quanto ao número de amigos, os estudos partem de díades (Vaughn, Colvin, Azria, Caya \& Krzysik, 2001), tríades (Lansford \& Parker, 1999) e chegam até redes de amigos (Salisch \& SeiffgeKrenke, 1996). Estas redes são investigadas em ambientes diversos, geralmente relacionadas ao ambiente escolar, mas também fora da escola (Smith, 1995) ou em diferentes ambientes (Ray, Cohen \& Secrist, 1995). Estas redes se compõem de formas diferentes, podendo incluir crianças populares e não populares (George \& Hartmann, 1996). Mesmo o impacto da tecnologia, como no caso da informática, nessas redes tem sido investigado (Griffiths, 1997). A amizade está relacionada tanto às características pessoais de cada participante, quanto às propriedades do relacionamento, desde aquele a dois até as redes mais complexas de amigos.

A família exerce diferentes influências sobre as relações de amizade na infância. Os pais afetam as amizades dos filhos por meio da estruturação de sua vida diária (Alles-Jardel, Fourdrinier, Roux \& Schneider, 2002), por meio de suas próprias amizades (Uhlendorff, 1996; Simpkins e Parke, 2001), através de seus conflitos (Kitzmann \& Cohen, 2003), da qualidade da relação entre pais e filhos (Freitag, Belsky, Grossmann, Grossmann \& Scheuerer-Englisch, 1996) e das propriedades do apego na infância (Chisholm, 1998). Os irmãos também exercem influência sobre as amizades (Cannoni, 2002). Diferentes orientações teóricas, como a teoria do apego e aprendizagem social, colaboram para a compreensão das relações entre amizade e família. De modo geral, a família é investigada como o contexto influenciando as amizades e não como parte da rede de amigos.

Amizades facilitam a adaptação da criança ao ambiente escolar e a aceitação pelos colegas de classe, o que depende diretamente de sua qualidade (Ladd, Kochenderfer \& Coleman, 1997), estando a presença de conflito na amizade associada a mau ajustamento, enquanto apoio percebido tem sido associado a melhores atitudes em relação à escola (Ladd, Kochenderfer \& Coleman, 1996). A amizade também facilita o ajustamento da criança tímida à escola, ao contribuir para a elevação de sua auto-estima e conseqüente proteção contra solidão e ansiedade, problemas internalizados decorrentes da timidez (Fordham \& Stevenson-Hinde, 1999). Crianças com amigos mostram um maior grau de ajuste à escola no início e no fim do ano escolar (Tomada, 2002) e a qualidade positiva das amizades 
no ambiente escolar também gera atitudes mais positivas em relação à escola (Alles-Jardel, Schneider \& Boutry, 2002). Finalmente, amizade e competência social têm sido investigados com vistas ao ajuste escolar. Alguns autores verificaram que crianças com amigos recíprocos possuem índices superiores de competência social quando comparadas a crianças sem amigos recíprocos. O número de amizades recíprocas também está associado com competência social. Nenhum benefício foi encontrado (em termos de competência social) para crianças que passam de uma série para a seguinte, na escola, acompanhada de um amigo (Vaughn \& cols, 2000). As amizades na escola contribuem para um melhor ajustamento da criança, além de trazer benefícios para o rendimento escolar.

\section{Amizades - Casos Especiais}

Os casos especiais incluem a amizade entre crianças com desenvolvimento atípico devido à presença de necessidades especiais (deficiência física ou mental) e os casos em que a criança enfrenta situações particulares, como ser vítima de abuso ou rejeição, estar enferma, ou em condições psicológicas alteradas, como no caso da depressão.

A amizade interfere diretamente na qualidade de vida da criança, inclusive a com necessidades especiais. A presença de deficiência física dificulta o estabelecimento e a manutenção de amizades devido a diversas limitações (Mulderij, 1997). Apesar das crianças típicas apresentarem atitudes favoráveis em relação a essas crianças, elas também percebem suas dificuldades de relacionamento (Tamm \& Prellwitz, 2001), cujos efeitos sociais são menores quando a deficiência física é temporária (Weiserbs \& Gottlieb, 2000). A deficiência mental também afeta as relações de amizade, apesar de ainda não se compreender claramente o papel da etiologia ou o tipo de deficiência (Freeman \& Kasari, 1998). Crianças típicas consideram difícil manter a amizade com portadores de deficiências devido a limitações na comunicação e problemas de comportamento (Lee, Yoo \& Bak, 2003). Amizades recíprocas envolvendo uma criança com problemas de aprendizagem apresentam menos comunicação (verbal e não-verbal) e colaboração na tomada de decisões, troca afetiva limitada, baixo nível de entrosamento na tarefa e distribuição hierárquica e assimétrica de papéis, comparáveis àquelas entre crianças de idades diferentes (Siperstein, Leffert \& Wenz-Gross, 1997).

Cada deficiência afeta a amizade de modo particular, mas, geralmente, não as impedem. Na Síndrome de Down, as amizades foram qualificadas como "emergentes", assemelhando-se aos relacionamentos precursores da amizade entre crianças típicas, seguindo cronologicamente as unilaterais e antecedendo as verdadeiras no desenvolvimento social (Freeman \& Kasari, 2002). Nas crianças autistas, as amizades são de qualidade inferior, faltando companheirismo, segurança, confiança e ajuda, trazendo menor satisfação (Bauminger \& Kasari, 2000). Crianças com deficiência de aprendizagem apresentaram menos amigos mútuos, mais amigos com déficit de aprendizagem, um número maior de amigos mais jovens e relacionamentos menos estáveis. Quanto à qualidade do relacionamento, houve mais conflito e problemas de reparo no relacionamento (Wiener \& Schneider, 2002).

Estratégias de facilitação de amizades entre crianças sem e com deficiência (atraso mental) foram observadas em mães destas crianças (Turnbull, Pereira \& Blue-Banning, 1999). Essas crianças apresentam relações limitadas com amigos em casa (Geisthardt, Brotherson \& Cook, 2002). Na escola, crianças em programas inclusivos apresentaram número de amigos semelhante às típicas quando as classes não concentravam crianças com deficiência (Buysse, Goldman \& Skinner, 2002). Crianças com necessidades especiais mantêm relações de amizade, apesar das dificuldades geradas por sua própria condição.

Os processos de rejeição (pelos pares) e abuso (por adultos), assim como os estados de depressão e solidão, afetam as amizades que, por sua vez, influenciam as consequiências destes processos e estados. Crianças rejeitadas pelos pares têm amizades, ainda que com qualidade inferior (Brendgen, Little \& Krappmann, 2000). A amizade também é um moderador dos efeitos da depressão e solidão (Martin, Cole, Clausen, Logan \& Strosher, 2003; Nangle, Erdley, Newman, Mason \& Carpenter, 2003), inclusive em crianças que sofreram abuso grave (Howe \& Parke, 2001) ou naquelas que são consideradas retraídas (Schneider, 1999; Doll, Murphy \& Song, 2003). Além disso, os amigos são informantes de sintomas depressivos de outra criança (Swenson \& Rose, 2003). A experiência de ter sofrido abuso afeta negativamente a amizade (Parker \& Herrera, 1996). Por sua vez, as amizades atenuam suas possíveis consequiências para a vida da criança. Do ponto de vista de saúde mental, a amizade parece ter efeitos positivos em crianças que sofreram abuso ou rejeição pelos pares e contribuem para a redução de estados de depressão e solidão. Por sua vez, rejeição, abuso, depressão e solidão também afetam o estabelecimento de amizades, dificultando, mas não impedindo, seu desenvolvimento. 


\section{CONSIDERAÇÕES FINAIS}

Na última década, uma diversidade de estudos contribuiu para a formação de um corpo de conhecimentos psicológicos sobre a amizade na infância, o qual se insere no campo mais amplo do relacionamento interpessoal. Além de um conjunto de procedimentos metodológicos relacionados ao estudo da amizade na infância, a literatura permite visualizar um conjunto teórico com três grandes áreas: a rede social e o contexto (particularmente o familiar e o escolar), as dimensões do relacionamento entre amigos (como similaridade, simetria, cooperação, competição, apoio social, conflito e agressividade) e os casos especiais, incluindo as relações de amizade de crianças com necessidades especiais, portadores de deficiências físicas e mentais, além daquelas de crianças enfrentando situações particulares, como enfermidades ou problemas psicológicos.

A literatura sobre os aspectos psicológicos da amizade na infância é marcada pela complexidade de temas e de perspectivas teóricas. Diferentes abordagens teóricas, procedimentos metodológicos e interesses práticos estão presentes. Por outro lado, a amizade na infância apresenta uma unidade ao se diferenciar de outras formas de relacionamentos, como a relação com pares ou colegas. Apesar dos avanços significativos, há várias possibilidades de pesquisa nas três áreas principais indicadas acima.

Quanto às amizades e às redes sociais, a sobreposição de relações interpessoais, como as relações de amizade e o relacionamento entre pais e filhos, é pouco investigada. Pouco se sabe sobre as semelhanças e diferenças entre as amizades típicas (com outra criança de idade próxima) e aquelas relatadas entre pais e filhos, ou entre as crianças e outras pessoas da família, como irmãos e primos. Crianças, por vezes, indicam um dos pais como amigo e chegam a indicá-lo como seu melhor amigo, mas a natureza desse relacionamento é pouco conhecida. A família tem sido investigada mais como contexto que influencia o desenvolvimento de aspectos relevantes para a amizade, como as relações de apego ou a influência de modelos parentais, do que como o palco de amizades.

Além do contexto familiar, a escola e a vizinhança também apresentam alguns tópicos pouco investigados. Alguns dados preliminares têm indicado um papel diferenciado da vizinhança na rede de amizades, dependendo do grupo social e do gênero. A rede de amizades fora da escola, em diferentes grupos sociais, pode revelar aspectos ainda pouco conhecidos da extensão e do significado dessas relações. As amizades na vizinhança apresentam mais possibilidades de hete- rogeneidade, maior participação de adultos e, possivelmente, uma maior diversidade de ambientes a serem explorados. Mesmo no ambiente escolar, há uma ênfase no papel da amizade para a adaptação do aluno. Pouco se sabe sobre o papel paralelo dos amigos na formação das crianças, por exemplo, quanto aos valores e à constituição de visão do mundo, em tópicos como informação social, sexual, cultural, entre outras. Como os amigos interferem na elaboração do conteúdo da informação transmitida pela escola também poderia ser mais bem explorado. Quanto a um contexto mais amplo, seria importante investigar como as amizades da criança se relacionam com os diferentes aspectos do contexto social, cultural, político e religioso.

Quanto às dimensões de análise, há algumas, tipicamente investigadas nos estudos sobre relacionamentos em geral, que ainda são pouco pesquisadas nas amizades da infância, como reciprocidade, intimidade, satisfação, auto-revelação, poder, interdependência, entre outras, que poderiam receber maior atenção. A compreensão da relação entre estas dimensões também poderia ser aprofundada. Outra limitação nos estudos empreendidos é a predominância de pesquisas transversais. Apesar das dificuldades de realização, estudos longitudinais permitiriam compreender melhor o início, o desenvolvimento (as transformações ao longo do tempo) e o término de amizades. Além disso, há pouca informação sobre a transição de uma amizade para outra e como amizades anteriores influenciam a constituição e as características de novas amizades. Em uma escala de tempo mais ampla, seria relevante investigar como as relações de amizade na infância afetam as amizades na adolescência, com efeitos significativos para a socialização do adolescente, afetando, inclusive, a tendência para o consumo de álcool e outras drogas e até o envolvimento com a criminalidade.

Os casos especiais de amizade, envolvendo pelo menos uma criança com desenvolvimento atípico, ou enfrentando uma situação especial, representam um amplo campo de investigação, com muitos pontos em aberto. Há uma grande diversidade de condições atípicas de desenvolvimento que carecem de estudos, assim como estados de enfermidade, no tocante à questão dos relacionamentos com amigos. Pouco se sabe sobre os diferentes tipos de deficiência ou enfermidades crônicas e seus efeitos sobre as propriedades da amizade. No sentido inverso, seria de interesse conhecer melhor o papel das amizades para o bem-estar e as condições de saúde da criança. Entre as condições associadas à enfermidade estão, entre outras, o isolamento (como no caso de hospitalização) da criança, os efeitos colaterais do tratamento (como na quimiotera- 
pia) e o prejuízo das condições de mobilidade (como fraturas ou problemas neurológicos).

A presente revisão da literatura identificou vários aspectos investigados nas amizades infantis. As contribuições são provenientes de diferentes abordagens ou enfatizam diferentes conceitos. Do ponto de vista de uma ciência do relacionamento, este quadro indica que, apesar da literatura sobre o tema ser relativamente extensa, podendo-se reconhecer um corpo de pesquisas concentradas neste campo de investigação, ela é de natureza dispersa, faltando uma maior integração visando à construção ou consolidação de uma "Psicologia da Amizade". No geral, o avanço da ciência do relacionamento interpessoal depende de pesquisa empírica e de avanços teóricos. No caso da investigação da amizade, há necessidade de avanço teórico visando à construção de um arcabouço teórico. Tal meta está em sintonia com propostas mais amplas, favoráveis à construção de uma ciência do relacionamento interpessoal (Hinde, 1997). A elaboração de modelos teóricos, específicos para a amizade, representa um desafio para o avanço da área e uma contribuição para a ciência do relacionamento interpessoal. Há a necessidade de modelos mais amplos, procurando integrar as dimensões estudadas em um sistema teórico comum. O diálogo entre as diferentes orientações teóricas deve ser estimulado, permitindo também um maior diálogo, integração e aperfeiçoamento das diversas estratégias metodológicas. Do ponto de vista geopolítico, faltam investigações em países em desenvolvimento, incluindo a América Latina. Os dados sobre os quais os trabalhos se baseiam são eminentemente norte-americanos e europeus. Há diferenças culturais, contudo não se sabe até que ponto a amizade de crianças latino-americanas, por exemplo, difeririam daquelas entre crianças de outras partes do mundo.

Além dos aspectos científicos, a importância social das amizades também deve ser levada em conta. As transformações sociais, culturais, políticas e econômicas abrem uma nova dimensão para as relações de amizade, desde a infância até a vida adulta. Fatores como alterações na estrutura familiar (famílias menores), maiores índices de escolarização e urbanização, o crescimento da inserção da mulher no mercado de trabalho e a convivência cada vez mais precoce da criança com seus pares em instituições sociais (desde as creches e escolas de educação infantil) resultam em uma maior intensidade e diversidade de contatos com estranhos, desde a infância, abrindo novas possibilidades e facilitando a formação de amizades. Do ponto de vista social, pode-se argumentar que as amizades carregam, em seu bojo, as sementes para a construção de uma sociedade democrática e igualitária, baseada na similaridade e em processos como cooperação e apoio social, realçando a importância do tema. Uma cultura da amizade é condizente com uma sociedade democrática, cuja conquista é uma das metas do mundo contemporâneo..

\section{REFERÊNCIAS}

Alles-Jardel, M., Fourdrinier, C., Roux, A. \& Schneider, B. H. (2002). Parents' structuring of children's daily lives in relation to the quality and stability of children's friendships. International Journal of Psychology, 37, 65-73.

Alles-Jardel, M., Schneider, B. H. \& Boutry, V. (2002). Friendship and attitudes toward school among children of two Muslim communities in Marseille. Early Education and Development, 13, 221-235.

Barry, C. McN. \& Wigfield, A. (2002). Self-perceptions of friendship-making ability and perceptions of friends' deviant behavior: childhood to adolescence. Journal of Early Adolescence, 22, 143-172.

Baumgartner, E. \& Camaioni, L. (1995). Le relazioni amicali nella prima infanzia. Costruzione e applicazione di una griglia di osservazione. Eta Evolutiva, 52, 21-33.

Bauminger, N. \& Kasari, C. (2000). Loneliness and friendship in high-functioning children with autism. Child Development, 71, 447-456.

Bensalah, L. (1995). Dyades asymetriques et relation amicale entre enfants. Enfance, 1, 53-69.

Bombi, A. S., Cannoni, E. \& Pinto, G. (2003). Le interviste silenziose: Studiare le relazioni amicali attraverso il disegno. Eta Evolutiva, 75, 97-103.

Booth, C. L., Rubin, K. H. \& Rose-Krasnor, L. (1998). Perceptions of emotional support from mother and friend in middle childhood: links with social-emotional adaptation and preschool attachment security. Child Development. 69, 427-442.

Brendgen, M., Bowen, F., Rondeau, N. \& Vitaro, F. (1999). Effects of friends' characteristics on children's social cognitions. Social Development, 8, 41-51.

Brendgen, M., Little, T. D. \& Krappmann, L. (2000). Rejected children and their friends: a shared evaluation of friendship quality? Merrill Palmer Quarterly, 46, 45-70.

Brendgen, M., Vitaro, F., Turgeon, L. \& Poulin, F. (2002). Assessing aggressive and depressed children's social relations with classmates and friends: a matter of perspective. Journal of Abnormal Child Psychology, 30, 609-624.

Brown, J. R., Donelan-McCall, N. \& Dunn, J. (1996). Why talk about mental states? The significance of children's conversations with friends, siblings, and mothers. Child Development, $67,836-849$.

Buysse, V., Goldman, B. D. \& Skinner, M. L. (2002). Setting effects on friendship formation among young children with and without disabilities. Exceptional Children, 68, 503-517.

Camaioni, L., Baumgartner, E. \& Perugini, M. (1998). Validazione di uno strumento per rilevare le relazioni amicali tra bambini nella prima infanzia. Giornale Italiano di Psicologia, 25, 101121. 
Cannoni, E. (2002). Amici e fratelli: Effetti dell'esperienza fraterna sulla rappresentazione di relazioni interpersonali infantili. Eta Evolutiva, 73, 70-77.

Chisholm, K. (1998). A three year follow-up of attachment and indiscriminate friendliness in children adopted from Romanian orphanages. Child Development, 69, 1092-1106.

Corsaro, W. A., Molinari, L., Hadley, K. G. \& Sugioka, H. (2003). Keeping and making friends: italian children's transition from preschool to elementary school. Social Psychology Quarterly, $66,272-292$

Doll, B. (1996). Children without friends: Implications for practice and policy. School Psychology Review, 25, 165-183.

Doll, B., Murphy, P. \& Song, S. Y. (2003). The relationship between children's self-reported recess problems, and peer acceptance and friendships. Journal of School Psychology, 41, 113-130.

Dunn, J. \& Cutting, A. L. (1999). Understanding others, and individual differences in friendship interactions in young children. Social Development, 8, 201-219.

Dunn, J., Cutting, A. L. \& Demetriou, H. (2000). Moral sensibility, understanding others, and children's friendship interactions in the preschool period. British Journal of Developmental Psychology, 18, 159-177.

Dunn, J., Cutting, A. L. \& Fisher, N. (2002). Old friends, new friends: predictors of children's perspective on their friends at school. Child Development, 73, 621-635.

Dunn, J. \& Hughes, C. (2001). "I got some swords and you're dead!": violent fantasy, antisocial behavior, friendship, and moral sensibility in young children. Child Development, 72, 491-505.

Erdley, C. A., Nangle, D. W. \& Gold, J. A. (1998). Operationalizing the construct of friendship among children: a psychometric comparison of sociometric-based definitional methodologies. Social Development, 7, 62-71.

Fonzi, A., Schneider, B. H., Tani, F. \& Tomada, G. (1997). Predicting children's friendship status from their dyadic interaction in structured situations of potential conflict. Child Development, 68, 496-506.

Fordham, K. \& Stevenson-Hinde, J. (1999). Shyness, friendship quality, and adjustment during middle childhood. Journal of Child Psychology and Psychiatry and Allied Disciplines, 40, 757-768.

Freeman, S. F. N. \& Kasari, C. (1998). Friendships in children with developmental disabilities. Early Education and Development, 9, 341-355.

Freeman, S. F. N. \& Kasari, C. (2002). Characteristics and qualities of the play dates of children with Down syndrome: Emerging or true friendships. American Journal on Mental Retardation, 107, 16-31.

Freitag, M. K., Belsky, J., Grossmann, K., Grossmann, K. E. \& Scheuerer-Englisch, H. (1996). Continuity in parent-child relationships from infancy to middle childhood and relations with friendship competence. Child Development, 67, 1437-1454.

French, D. C., Jansen, E. A., Riansari, M. \& Setiono, K. (2003). Friendships of indonesian children: adjustment of children who differ in friendship presence and similarity between mutual friends. Social Development, 12, 606-621.
French, D. C., Rianasari, M., Pidada, S., Nelwan, P. \& Buhrmester, D. (2001). Social support of Indonesian and U.S. children and adolescents by family members and friends. Merrill Palmer Quarterly, 47, 377-394.

Geisthardt, C. L., Brotherson, M. J. \& Cook, C. C. (2002). Friendships of children with disabilities in the home environment. Education and Training in Mental Retardation and Developmental Disabilities, 37, 235-252.

George, T. P. \& Hartmann, D. P. (1996). Friendship networks of unpopular, average, and popular children. Child Development, 67, 2301-2316.

Gifford-Smith, M. E. \& Brownell, C. A. (2003). Childhood peer relationships: social acceptance, friendships, and peer networks. Journal of School Psychology, 41, 235-284.

Graham, J. A. \& Cohen, R. (1997). Race and sex as factors in children's sociometric ratings and friendship choices. Social Development, 6, 355-372.

Graham, J. A., Cohen, R., Zbikowski, S. M. \& Secrist, M. E. (1998). A longitudinal investigation of race and sex as factors in children's classroom friendship choices. Child Study Journal, 28, 245-266.

Griffiths, M. (1997). Friendship and social development in children and adolescents: The impact of electronic technology. Educational and Child Psychology, 14(3), 25-37.

Halle, T. G. (1999). Implicit theories of social interactions: Children's reasoning about the relative importance of gender and friendship in social partner choices. Merrill Palmer Quarterly, 45, 445-467.

Haselager, G. J. T., Hartup, W. W., Lieshout, C. F. M. van \& Riksen-Walraven, J. M. A. (1998). Similarities between friends and nonfriends in middle childhood. Child Development, 69, $1198-1208$

Hawley, P. H., Little, T. D. \& Pasupathi, M. (2002). Winning friends and influencing peers: Strategies of peer influence in late childhood. International Journal of Behavioral Development, 26, 466-474.

Hinde, R. A. (1997). Relationships: A Dialectical Perspective. Hove: Psychology Press.

Hortacsu, N., Gencoez, T. \& Oral, A. (1995). Perceived functions of family and friends during childhood, adolescence, and youth: Developmental theories of two Turkish groups. International Journal of Psychology, 30, 591-606.

Howe, T. R. \& Parke, R. D. (2001). Friendship quality and sociometric status: between-group differences and links to loneliness in severely abused and nonabused children. Child Abuse and Neglect, 25, 585-606.

Hughes, C., Cutting, A. L. \& Dunn, J. (2001). Acting nasty in the face of failure? Longitudinal observations of "hard-to-manage" children playing a rigged competitive game with a friend. Journal of Abnormal Child Psychology, 29, 403-416.

Hundley, R. J. \& Cohen, R. (1999). Children's relationships with classmates: a comprehensive analysis of friendship nominations and liking. Child Study Journal, 29, 233-246.

Kitzmann, K. M. \& Cohen, R. (2003). Parents'versus children's perceptions of interparental conflict as predictors of children's friendship quality. Journal of Social and Personal Relationships, 20, 689-700. 
Kupersmidt, J. B., DeRosier, M. E. \& Patterson, C. P. (1995) Similarity as the basis for children's friendships: The roles of sociometric status, aggressive and withdrawn behavior, academic achievement and demographic characteristics. Journal of Social and Personal Relationships, 12, 439-452.

Ladd, G. W., Kochenderfer, B. J. \& Coleman, C. C. (1996). Friendship quality as a predictor of young children's early school adjustment. Child Development, 67, 1103-1118.

Ladd, G. W., Kochenderfer, B. J. \& Coleman, C. C. (1997). Classroom peer acceptance, friendship, and victimization: distinct relational systems that contribute uniquely to children's school adjustment? Child Development, 68, 1181-1197.

Lansford, J. E. \& Parker, J. G. (1999). Children's interactions in triads: behavioral profiles and effects of gender and patterns of friendships among members. Developmental Psychology, 35, 80-93.

Lee, S. H., Yoo, S. Y. \& Bak, S. H. (2003). Characteristics of friendships between children with and without mild disabilities. Education and Training in Developmental Disabilities, 38, 157-166.

Linsey, E. W. (2002). Preschool children's friendships and peer acceptance: Links to social competence. Child Study Journal, $32,145-156$.

Little, T. D., Brendgen, M., Wanner, B. \& Krappmann, L. (1999). Children's reciprocal perceptions of friendship quality in the sociocultural contexts of East and West Berlin. International Journal of Behavioral Development, 23, 63-89.

Maguire, M. C. \& Dunn, J. (1997). Friendships in early childhood, and social understanding. International Journal of Behavioral Development, 21, 669-686.

Martin, J. M., Cole, D. A., Clausen, A., Logan, J. \& Strosher, H. L. W. (2003). Moderators of the relation between popularity and depressive symptoms in children: processing strength and friendship value. Journal of Abnormal Child Psychology, 31, 471-483.

McElwain, N. L. \& Volling, B. L. (2002). Relating individual control, social understanding, and gender to child-friend interaction: a relationships perspective. Social Development, 11, 362-385.

Meurling, C. J. N., Ray, G. E. \& LoBello, S. G. (1999). Children's evaluations of classroom friend and classroom best friend relationships. Child Study Journal, 29, 79-96.

Miell, D. \& MacDonald, R. (2000). Children's creative collaborations: The importance of friendship when working together on a musical composition. Social Development, 9, 348-369.

Mulderij, K. J. (1997). Peer relations and friendship in physically disabled children. Child: Care, Health and Development, 23, 379-389.

Nangle, D. W., Erdley, C. A., Newman, J. E., Mason, C. A. \& Carpenter, E. M. (2003). Popularity, friendship quantity, and friendship quality: interactive influences on children's loneliness and depression. Journal of Clinical Child and Adolescent Psychology, 32, 546-555.

Ollendick, T. H., Yang, B., Dong, Q., Xia, Y. \& Lin, L. (1995). Perceptions of fear in other children and adolescents: the role of gender and friendship status. Journal of Abnormal Child Psychology, 23, 439-452.
Parker, J. G. \& Herrera, C. (1996). Interpersonal processes in friendship: a comparison of abused and nonabused children's experiences. Developmental Psychology, 32, 1025-1038.

Parker, J. G. \& Seal, J. (1996). Forming, losing, renewing, and replacing friendships: applying temporal parameters to the assessment of children's friendship experiences. Child Development, 67, 2248-2268.

Phillipsen, L. C., Deptula, D. P. \& Cohen, R. (1999). Relating characteristics of children and their friends to relational and overt aggression. Child Study Journal, 29, 269-289.

Pinto, G., Bombi, A. S. \& Cordioli, A. (1997). Similarity of friends in three countries: a study of children's drawings. International Journal of Behavioral Development, 20, 453-469.

Poulin, F., Cillessen, A. H. N., Hubbard, J. A., Coie, J. D., Dodge, K. A. \& Schwartz, D. (1997). Children's friends and behavioral similarity in two social contexts. Social Development, 6, 224236.

Prinstein, M. J., La-Greca, A. M., Vernberg, E. M. \& Silverman, W. K. (1996). Children's coping assistance: how parents, teachers and friends help children cope after a natural disaster. Journal of Clinical Child Psychology, 25, 463-475.

Ray, G. E. \& Cohen, R. (1996). Children's friendships: Expectations for prototypical versus actual best friends. Child Study Journal, 26, 209-227.

Ray, G. E., Cohen, R. \& Secrist, M. E. (1995). Best friend networks of children across settings. Child Study Journal, 25, 169188.

Ray, G. E., Cohen, R., Secrist, M. E. \& Duncan, M. K. (1997). Relating aggressive and victimization behaviors to children's sociometric status and friendships. Journal of Social and Personal Relationships, 14, 95-108.

Rizzo, T. A. \& Corsaro, W. A. (1995). Social support processes in early childhood friendship: a comparative study of ecological congruences in enacted support. American Journal of Cоттиnity Psychology, 23, 389-417.

Rose, A. J. \& Asher, S. R (1999). Children's goals and strategies in response to conflicts within a friendship. Developmental Psychology, 35(1), 69-79.

Rotenberg, K. J. (1995). Development of children's restrictive disclosure to friends. Journal of Genetic Psychology, 156, 279292.

Rotenberg, K. J. \& Morgan, C. J. (1995). Development of a scale to measure individual differences in children's trust-value basis of friendship. Journal of Genetic Psychology, 156, 489-502.

Salisch, M. von (2001). Children's emotional development: challenges in their relationships to parents, peers, and friends. International Journal of Behavioral Development, 25, 310-319.

Salisch, M. von \& Seiffge-Krenke, I. (1996). Freundschaften im Kindes- und Jugendalter: Konzepte, Netzwerke, Elterneinfluesse. Psychologie in Erziehung und Unterricht, 43, 85-99.

Schneider, B. H. (1999). A multimethod exploration of the friendships of children considered socially withdrawn by their school peers. Journal of Abnormal Child Psychology, 27, 115-123.

Schneider, B. H., Fonzi, A., Tani, F. \& Tomada, G. (1997). A cross-cultural exploration of the stability of children's friendships and predictors of their continuation. Social Development, $6,322-339$ 
Schneider, B. H. \& Greenman, P. S. (2003). Misurare le amicizie dei bambini: le sfide nell'accedere ad uno stato co-costruito della mente. Eta Evolutiva, 75, 70-78.

Sebanc, A. M. (2003). The friendship features of preschool children: links with prosocial behavior and aggression. Social Development, 12, 249-268.

Simpkins, S. D. \& Parke, R. D. (2001). The relations between parental friendships and children's friendships: self-report and observational analysis. Child Development, 72, 569-582.

Simpkins, S. D. \& Parke, R. D. (2002). Do friends and nonfriends behave differently? A social relations analysis of children's behavior. Merrill Palmer Quarterly, 48, 263-283.

Siperstein, G. N., Leffert, J. S. \& Wenz-Gross, M. (1997). The quality of friendships between children with and without learning problems. American Journal on Mental Retardation, 102, 111-125.

Slomkowski, C. \& Dunn, J. (1996). Young children's understanding of other people's beliefs and feelings and their connected communication with friends. Developmental Psychology, 32, 442-447.

Smith, M. C. (1995). A preliminary description of nonschoolbased friendship in young high-risk children. Child Abuse and Neglect, 19, 1497-1511.

Smorti, A. \& Pagnucci, S. (2003). Le parole dell'amicizia: studiare le relazioni amicali attraverso le storie. Eta Evolutiva, 75, 8896.

Sturgess, W., Dunn, J. \& Davies, L. (2001). Young children's perceptions of their relationships with family members: links with family setting, friendships, and adjustment. International Journal of Behavioral Development, 25, 521-529.

Swenson, L. P. \& Rose, A. J. (2003). Friends as reporters of children's and adolescents' depressive symptoms. Journal of Abnormal Child Psychology, 31, 619-631.

Tamm, M. \& Prellwitz, M. (2001). "If I had a friend in a wheelchair": Children's thoughts on disabilities. Child: Care, Health and Development, 27, 223-240.

Tani, F. (2000). Le amicizie negli anni della scuola: Un'indagine sulle caratteristiche personali dei bambini con molti amici. Eta Evolutiva, 67, 52-59.

Tomada, G. (2000). Il ruolo della contrapposizione nel mantenimento della relazione amicale in eta prescolare e scolare. Eta Evolutiva, 67, 15-24.
Tomada, G. (2002). Disagio scolastico e bambini senza amici. Eta Evolutiva, 71, 92-98.

Turnbull, A. P., Pereira, L. \& Blue-Banning, M. J. (1999). Parents' facilitation of friendships between their children with a disability and friends without a disability. Journal of the Association for Persons with Severe Handicaps, 24(2), 85-99.

Uhlendorff, H. (1996). Elterliche soziale Netzwerke in ihrer Wirkung auf die Freundschaftsbeziehungen der Kinder. Psychologie in Erziehung und Unterricht, 43, 127-140.

Vaughn, B. E., Azria, M. R., Krzysik, L., Caya, L. R., Bost, K. K., Newell, W. \& Kazura, K. L. (2000). Friendship and social competence in a sample of preschool children attending Head Start. Developmental Psychology, 36, 326-338.

Vaughn, B. E., Colvin, T. N., Azria, M. R., Caya, L. \& Krzysik, L. (2001). Dyadic analyses of friendship in a sample of preschoolage children attending Head Start: correspondence between measures and implications for social competence. Child Development, 72, 862-878.

Wallace, J. R. \& Almy, T. L. (1999). Do children's maps differ for younger and older friends? Journal of Genetic Psychology, $160,89-98$.

Weiserbs, B. \& Gottlieb, J. (2000). The effect of perceived duration of physical disability on attitudes of school children toward friendship and helping. Journal of Psychology, 134, 343345.

Weiss, M. R., Smith, A. L. \& Theeboom, M. (1996). "That's what friends are for": children's and teenagers' perceptions of peer relationships in the sport domain. Journal of Sport and Exercise Psychology, 18, 347-379.

Wiener, J. \& Schneider, B. H. (2002). A multisource exploration of the friendship patterns of children with and without learning disabilities. Journal of Abnormal Child Psychology, 30, 127141.

Yugar, J. M. \& Shapiro, E. S. (2001). Elementary children's school friendship: a comparison of peer assessment methodologies. School Psychology Review, 30, 568-585.

Recebido: $14 / 09 / 2005$

Revisado: $13 / 12 / 2005$

Aceito: $21 / 12 / 2005$

\section{Sobre o autor:}

Agnaldo Garcia: Doutor em Psicologia pela Universidade de São Paulo.

Endereço para correspondência: Av. Des. Cassiano Castelo, 369 - 29173-037 - Manguinhos - Serra/ES (agnaldo.garcia@uol.com.br) 